Lin, P. P. and Datseris, P., 1986, "Development of a Position and Force Sensor for Robotic Applications," Proceedings of 1986 IEEE International Conference on Robotics and Automation, Vol. 3, Apr. 7-10, pp. 1798-1805.

Nadai, A., 1915, "Die Formanderungen und die Spannungen von Rechteckigen Elastichen Pattern," Forsch A. D. Gebiete D. Ingenieurwesens, Berlin, Nos. 170 and 171.

Szilard, R., 1974, Theory and Analysis of Plates, New Jersey, Prentice Hall, pp. $36-42$.

Timoshenko, S. and Woinowsky-Krieger, S., 1959, Theory of Plates and Shells, Second Edition, McGraw-Hill, New York, pp. 113-114.

\section{Unstable Elastic Materials and the Viscoelastic Response of Bars in Tension ${ }^{5}$}

\section{R. Abeyaratne ${ }^{6}$ and J. K. Knowles ${ }^{7}$}

Some homogeneous elastic materials are capable of sustaining finite equilibrium deformations with discontinuous strains. For materials of this kind, the energetics of isothermal, quasi-static motions may differ from those conventionally associated with elastic behavior. When equilibrium states involving strain jumps occur during such motions, the rate of increase of stored energy in a portion of the body may no longer coincide with the rate of work of the external forces present. In general, energy balance now includes an additional effect due to the presence of moving strain discontinuities. As a consequence, the macroscopic response of the body may be dissipative. This fact makes it possible to model certain types of inelastic behavior in solids with the help of such "unstable" elastic materials; see, for example, Abeyaratne and Knowles (1987a,b,c).

The purpose of the present note is to illustrate behavior of this kind with the help of an especially simple example involving the extensional deformations of a bar treated as a onedimensional continuum. The bar is composed of an unstable elastic material of the type considered by Ericksen (1975). We show that the force-elongation relation (or "macroscopic response") of the bar during a quasi-static motion may be viscoelastic when a moving strain jump is present, even though the underlying constitutive law is elastic in the sense that, at each particle, the present stress is determined by the present strain.

Consider an elastic bar which, in the reference configuration, occupies the interval $[0, L]$ of the $x$-axis and has constant cross-sectional area $A$. In a deformation, the particle at $x$ is carried to $y=x+u(x)$, where $u$ is the displacement. We assume that $u$ is continuous on $[0, L]$, and that $u^{\prime}(x)$ exists and is continuous for $0 \leq x \leq s$ and $s \leq x \leq L$, but $u^{\prime}(s+)$ and $u^{\prime}(s-)$ may differ; here $0<s<L$. If $x \neq s$, the strain at $x$ is $\epsilon(x)=u^{\prime}(x)$. It is required that $\epsilon(x)>-1$ for $x \neq s$, so that the mapping $x \rightarrow y$ is invertible. We take the left end of the bar to be fixed, so that $u(0)=0$.

Suppose that the material is elastic with strain energy per unit reference volume $W(\epsilon)$. The nominal stress response function is then

$$
\hat{\sigma}(\epsilon)=W^{\prime}(\epsilon),
$$

so that the nominal stress in the bar at particle $x$ is $\sigma(x)=\hat{\sigma}(\epsilon(x))$. We shall be concerned with the special "trilinear" stress response function given by

\footnotetext{
${ }^{5}$ The results reported here were obtained in the course of an investigation supported in part by the U.S. Office of Naval Research under Contract N00014-87-K-0117.

${ }^{6}$ Department of Mechanical Engineering, Massachusetts Institute of Technology, Cambridge, MA 02139.

Division of Engineering and Applied Science, California Institute of Technology, Pasadena, CA 91125. Manuscript received by ASME Applied Mechanics Division, November 10 ,
1987 .
}

The graph of $\hat{\sigma}(\epsilon)$ is shown in Fig. 1, where the meanings of the constants $\mu, \epsilon_{M}, \epsilon_{m}$ and $\epsilon_{0}=\epsilon_{M}+\epsilon_{m}$ are made clear. One may think of the material as exhibiting three phases: the first phase is represented by the rising branch of the stress-strain curve through the origin, the third phase corresponds to the final rising branch, and the declining portion of the curve represents an unstable intervening second phase. In the present case, the first and third phases are both associated with the same modulus $\mu$.

In the absence of body force, the bar will be in equilibrium if the nominal stress $\sigma(x)$ satisfies

$$
\sigma(x)=\sigma=\text { constant, } 0 \leqq x \leqq L .
$$

A deformation with a strain discontinuity of the kind described above will correspond to an equilibrium state if it is of the form

$$
u(x)=\left\{\begin{array}{l}
\epsilon_{1} x, \quad 0 \leqq x \leqq s, \\
\epsilon_{2} x+\left(\epsilon_{1}-\epsilon_{2}\right) s, \quad s \leqq x \leqq L,
\end{array}\right.
$$

where the strains $\epsilon_{1}$ and $\epsilon_{2}$ are constant and such that

$$
\hat{\sigma}\left(\epsilon_{1}\right)=\hat{\sigma}\left(\epsilon_{2}\right)=\sigma \text {. }
$$

We shall be concerned here only with those deformations of the form (4) in which

$$
-\epsilon_{M} \leqq \epsilon_{1} \leqq \epsilon_{M}, \epsilon_{m} \leqq \epsilon_{2} \leqq \epsilon_{M}+\epsilon_{0},
$$

so that only phases one and three are represented. For an equilibrium state of this kind, the total energy stored in the bar is

$$
E=A W\left(\epsilon_{1}\right) s+A W\left(\epsilon_{2}\right)(L-s) .
$$

Now suppose that the bar is in a quasi-static motion during which, at each instant $t$, the displacement $u(x, t)$ is of the form (4), with $s=s(t), \epsilon_{1}=\epsilon_{1}(t)$ and $\epsilon_{2}=\epsilon_{2}(t)$. The restrictions (5) and (6) are to hold for all $t$, with $\sigma=\sigma(t)$. Assume that $s(t)$, $\epsilon_{1}(t)$ and $\epsilon_{2}(t)$ are continuous and piecewise continuously differentiable in $t$. During such a motion, the energy $E(t)$ is given by (7) with $\epsilon_{1}, \epsilon_{2}$, and $s$ now functions of time. A direct calculation gives

$$
\dot{E}(t)=F(t) \dot{\delta}(t)+A[-f(t)] \dot{s}(t),
$$

where $F(t)=\sigma(t) A$ is the force,

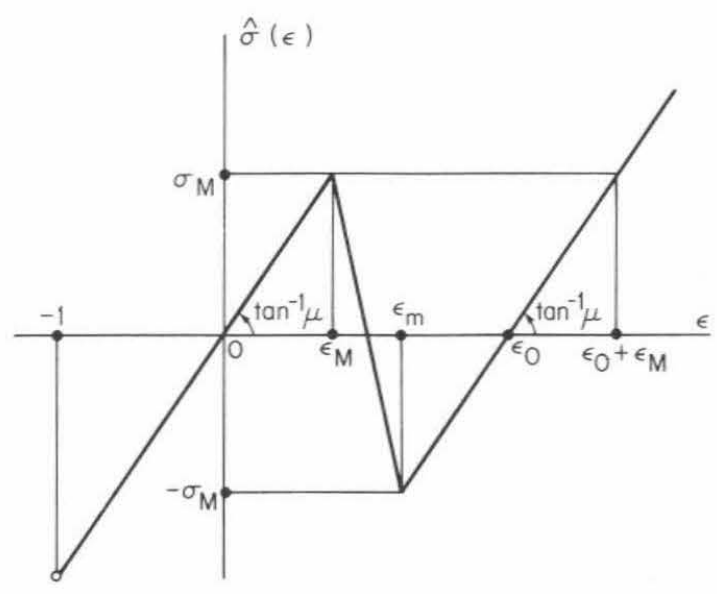

Fig. 1 Stress response function 


$$
\delta(t)=u(L, t)=\epsilon_{1}(t) s(t)+\epsilon_{2}(t)(L-s(t))
$$

is the elongation of the bar at time $t$, and $f(t)$ is given by

$$
f(t)=W\left(\epsilon_{2}(t)\right)-W\left(\epsilon_{1}(t)\right)-\sigma(t)\left(\epsilon_{2}(t)-\epsilon_{1}(t)\right) .
$$

The first term on the right in (8) is the rate of work of the force acting on the end $x=L$ of the bar. The second term may be interpreted as the rate of work done by a fictitious "traction" $f(t)$ in moving the strain discontinuity at $x=s(t)$. For the stress-strain relation (2), one finds using (1), (6) and (10) that

$$
f(t)=-\epsilon_{0} \sigma(t) \text {. }
$$

If the motion takes place isothermally, the second law of thermodynamics requires that $\dot{E}-F \dot{\delta}$ be nonnegative; thus the motion must be such that

$$
f(t) \dot{s}(t) \geqq 0 .
$$

Because of (5) and (6), specifying the history of the stress acting on the bar determines $\epsilon_{1}(t)$ and $\epsilon_{2}(t)$, but leaves undetermined the location $s(t)$ of the strain jump, and therefore the elongation history $\delta(t)$ of (9) as well.

In formulas (7) and (9), one may regard $s$ as an "internal variable" akin to those arising in microstructural theories of plasticity; see, for example, Rice (1971). This suggests that, by analogy with such theories, the constitutive description of the material should be augmented by relating the fictitious traction $f(t)$ to the velocity of the moving discontinuity $\dot{s}(t)$. One form that such a "kinetic relation" might take is

$$
\dot{s}(t)=V(f(t)),
$$

where $V$ is a function determined by the material. Using (11), one infers from (13) that

$$
\dot{s}(t)=V\left(-\epsilon_{0} \sigma(t)\right) \text {. }
$$

Note from (12) that every admissible $V$ must be such that $V(f) f \geqq 0$.

Let $\gamma(t)=\delta(t) / L$ be the relative elongation of the bar. The relation between $\gamma$ and $F$-the macroscopic response of the bar-may now be determined as follows. Using (2) and (6) in (9) yields

$$
\delta(t)=\epsilon_{0}[L-s(t)]+\sigma(t) L / \mu,
$$

from which, with the help of (14), one finds the nonlinear viscoelastic relation

$$
\dot{\gamma}(t)=\dot{F}(t) / \mu A-\epsilon_{0} V\left(-\epsilon_{0} F(t) / A\right) / L .
$$

If in particular the kinetic response function $V$ is specified through $V(f)=f / \nu$, where $\nu$ is a positive constant, (13) provides a linearly "viscous" kinetic relation between $f$ and $\dot{s}$. The macroscopic response relation (16) specializes to

$$
\dot{\gamma}(t)=\dot{F}(t) / \mu A+\epsilon_{0}^{2} F(t) / \nu L A .
$$

This is precisely the form of the response relation characteristic of the so-called "Maxwell" spring-dashpot model of elementary viscoelasticity. Other forms of macroscopic response can be obtained by replacing (13) by a more general kinetic relation.

A more extensive discussion of the one-dimensional theory of quasi-static motions of bars composed of unstable elastic materials may be found in Abeyaratne and Knowles (1987c).

\section{References}

Abeyaratne, R. and Knowles, J. K., 1987a, "Non-elliptic Elastic Materials and the Modeling of Elastic-plastic Behavior for Finite Deformation," Journal of the Mechanics and Physics of Solids, Vol. 35, pp. 343-365.

Abeyaratne, R. and Knowles, J. K., 1987b, "Non-elliptic Elastic Materials and the Modeling of Dissipative Mechanical Behavior: An Example," Journal of Elasticity, Vol. 18, pp. 227-278.

Abeyaratne, R. and Knowles, J. K., 1987c, "On the Dissipative Response due to Discontinuous Strains in Bars of Unstable Elastic Material," Technical Report No. 1, ONR Contract N00014-87-K-0117, California Institute of Technology, September, 1987; to appear in International Journal of Solids and Structures.
Ericksen, J. L., 1975, "Equilibrium of Bars," Journal of Elasticity, Vol. 5, pp. 191-201.

Rice, J. R., 1971, "Inelastic Constitutive Relations for Solids: An Internal Variable Theory and its Application to Metal Plasticity," Journal of the Mechanics and Physics of Solids, Vol. 19, pp. 433-455.

\section{Diffusion of Self-Equilibrating End Loads in Elastic Solids}

\section{Durban ${ }^{8}$ and W. J. Stronge ${ }^{9}$}

\section{Introduction}

The classical Papkovich-Fadle eigenfunction analysis for axial diffusion of self-equilibrating end loads, in plane strain linear elasticity, predicts exponential decay of the form exp $(-k z / h)$ where $z \geq 0$ is the axial coordinate, $h$-half thickness, and $k$ is any root of the associated eigenvalue equation. The lowest rate of axial decay for an elastic solid at small strains is governed by the first symmetric eigenfunction with $k_{1}=2.1061+i 1.1254$. The next term, in order of increasing magnitude of $\operatorname{Re}\{k\}$, is given by the first antisymmetric eigenfunction with $k_{2}=3.7488+i 1.3843$. These values of $k$, as well as the higher roots, are determined by the transcendental equations $\sin 2 k \pm 2 k=0$ where the $(+)$ and $(-)$ signs correspond to symmetric and antisymmetric eigenfunctions, respectively.

A recent paper by Abeyaratne, Horgan and Chung (1985) has addressed the plane strain end effect problem within the framework of finite strain elasticity. The authors consider an incrementally self-equilibrating end disturbance imposed on a uniformly stretched strip. The axial decay rate is again exponential but the eigenvalue $k$ is now dependent on the magnitude of initial stretch. Furthermore, the variation of $k$ with the initial stretch displays considerable sensitivity to the form of the strain energy function. Unfortunately, that study has been restricted to the subspace of symmetric eigenfunctions so it reveals only part of the complete picture.

The main purpose of the present note is to demonstrate that the smallest decay rate (which provides a bound on the validity of Saint-Venant's principle) can be associated with the first antisymmetric eigenfunction. It is necessary, therefore, to examine the complete space of eigenfunctions (i.e., symmetric and antisymmetric) allowing for competition among the corresponding eigenvalues over the entire range of prestrain and material parameters.

The basic setting is similar to that of Abeyaratne, Horgan and Chung (1985), though we differ in the solution method and follow the Hill and Hutchinson (1975) stream function formulation. The material is assumed to be hyperelastic and incompressible. Specific examples are illustrated for the Mooney-Rivlin model and for a power-law solid. A few related topics are discussed and some general results are presented.

\section{Formulation of the Problem}

A semi-infinite strip of rectangular cross section (Fig. 1) is uniformly stretched under plane strain conditions. The current axial stress is $\sigma_{z}=\sigma$ and the principal stretches are $\lambda_{z}=\lambda$ and $\lambda_{x}=\lambda^{-1}$. The deformed thickness is denoted by $2 h$ and the faces $x= \pm h$ are stress free. Note that $h=H / \lambda$ where $H$ is the original undeformed thickness.

\footnotetext{
${ }^{8}$ Department of Aeronautical Engineering, Technion, Haifa 32000, Israel.

9 Department of Engineering, University of Cambridge, Trumpington Street, Cambridge CB2 1PZ, England.

Manuscript received by ASME Applied Mechanics Division, August 25, 1987.
} 